\title{
BMJ Open Exploring the role of pain as an early predictor of category 2 pressure ulcers: a prospective cohort study
}

\author{
Isabelle L Smith, ${ }^{1}$ Sarah Brown, ${ }^{1}$ Elizabeth McGinnis, ${ }^{1,2}$ Michelle Briggs, ${ }^{3}$ \\ Susanne Coleman, ${ }^{1}$ Carol Dealey, ${ }^{4}$ Delia Muir, ${ }^{1} \mathrm{E}$ Andrea Nelson, ${ }^{5}$ \\ Rebecca Stevenson, ${ }^{6}$ Nikki Stubbs, ${ }^{7}$ Lyn Wilson, ${ }^{1,8}$ Julia M Brown, ${ }^{1}$ Jane Nixon ${ }^{1}$
}

To cite: Smith IL, Brown S, McGinnis E, et al. Exploring the role of pain as an early predictor of category 2 pressure ulcers: a prospective cohort study. BMJ Open 2017;7:e013623.

doi:10.1136/bmjopen-2016013623

- Prepublication history for this paper is available online. To view these files please visit the journal online (http://dx.doi.org/10.1136/ bmjopen-2016-013623).

Received 26 July 2016 Revised 3 November 2016 Accepted 2 December 2016

CrossMark

For numbered affiliations see end of article.

Correspondence to Professor Jane Nixon; j.e.nixon@leeds.ac.uk

\section{ABSTRACT}

Objective: To explore pressure area related pain as a predictor of category $\geq 2$ pressure ulcer (PU)

development.

Design: Multicentre prospective cohort study.

Setting: UK hospital and community settings.

Participants inclusion: Consenting acutely ill patients aged $\geq 18$ years, defined as high risk (Braden bedfast/chairfast AND completely immobile/very limited mobility; pressure area related pain or; category $1 \mathrm{PU}$ ).

Exclusion: Patients too unwell, unable to report pain, 2 or more category $\geq 2$ PUs.

Follow-up: Twice weekly for 30 days.

Primary and secondary outcome measures:

Development and time to development of one or more category $\geq 2$ PUs.

Results: 0 f 3819 screened, 1266 were eligible, 634 patients were recruited, 32 lost to follow-up, providing a 602 analysis population. $152(25.2 \%)$ developed one or more category $\geq 2$ PUs. $464(77.1 \%)$ patients reported pressure area related pain on a healthy, altered or category 1 skin site of whom $130(28.0 \%)$ developed a category $\geq 2$ PU compared with $22(15.9 \%)$ of those without pain. Full stepwise variable selection was used throughout the analyses. (1) Multivariable logistic regression model to assess 9 a priori factors: presence of category $1 \mathrm{PU}(\mathrm{OR}=3.25,95 \% \mathrm{Cl}(2.17$ to 4.86$)$, $p<0.0001)$, alterations to intact skin $(\mathrm{OR}=1.98,95 \% \mathrm{Cl}$ (1.30 to 3.00), $p=0.0014$ ), pressure area related pain ( $O R=1.56,95 \% \mathrm{Cl}$ (0.93 to 2.63), $p=0.0931)$. (2) Multivariable logistic regression model to account for overdispersion: presence of category $1 \mathrm{PU}(\mathrm{OR}=3.20$, $95 \% \mathrm{Cl}$ (2.11 to 4.85), $p<0.0001$ ), alterations to intact skin $(\mathrm{OR}=1.90,95 \% \mathrm{Cl}$ (1.24 to 2.91), $\mathrm{p}=0.0032)$, pressure area related pain $(\mathrm{OR}=1.85,95 \% \mathrm{Cl}(1.07$ to $3.20), p=0.0271)$, pre-existing category $2 \mathrm{PU}(\mathrm{OR}=2.09$, $95 \% \mathrm{Cl}$ (1.35 to 3.23$), p=0.0009)$, presence of chronic wound (OR=1.66, $95 \% \mathrm{Cl}$ (1.06 to 2.62), $p=0.0277$ ), Braden activity $(p=0.0476)$. (3) Accelerated failure time model: presence of category $1 \mathrm{PU}(\mathrm{AF}=2.32,95 \% \mathrm{Cl}$ (1.73 to 3.12$), p<0.0001$ ), pressure area related pain $(A F=2.28,95 \% \mathrm{Cl}$ (1.59 to 3.27), $p<0.0001)$. (4) 2-level random-intercept logistic regression model: skin status which comprised 2 levels (versus healthy skin); alterations to intact skin $(\mathrm{OR}=4.65,95 \% \mathrm{Cl}(3.01$ to 7.18), $p<0.0001)$, presence of category $1 \mathrm{PU}$

\section{Strengths and limitations of this study}

- The study was designed to incorporate key quality criteria for the conduct and reporting of risk factor/prognostic factor studies to promote generalisability and minimise bias.

- The primary outcome, the development of new category $\geq 2$ pressure ulcers (PUs), provides the most reliable outcome measure of PU development.

- Patients were recruited from hospital and community settings and are representative of UK 'standard care'.

- There was no blinded outcome assessment due to funding and/or capacity within the clinical research teams.

- It is acknowledged that the patient population is not representative of the general NHS population through exclusion of patients who had cognition problems, were very sick or were terminally ill.

(OR=17.30, 95\% Cl (11.09 to 27.00), $\mathrm{p}<0.0001$ ) and pressure area related pain $(\mathrm{OR}=2.25,95 \% \mathrm{Cl}(1.53$ to 3.29), $p<0.0001$ ).

Conclusions: This is the first study to assess pain as a predictor of category $\geq 2 \mathrm{PU}$ development. In all 4 models, pain emerged as a risk factor associated with an increased probability of category $\geq 2$ PU development.

\section{INTRODUCTION}

\section{Background}

A systematic review of health-related quality of life in patients with pressure ulcers (PUs) identified that patients reported experiencing pain at 'pressure areas' prior to clinical PU manifestation but that their reports of pain were ignored. Patients blamed healthcare professionals when a PU developed subsequently, due to the lack of action. ${ }^{1}$ A PU risk factor systematic review did not identify any studies which included pain as a candidate risk factor in multivariable analysis, ${ }^{2}$ and 
previous cross-sectional studies have focused on pain associated with existing category $\geq 2$ PUs. ${ }^{3-5}$

As part of the National Institute for Health Research (NIHR) funded PU Programme of Research (PURPOSE) ${ }^{6}$ we first sought to determine the extent of pain experienced by patients with intact skin on pressure areas. ${ }^{78}$ We conducted two large multicentre prevalence surveys in the hospital ${ }^{8}$ and community ${ }^{7}$ setting. It was identified that $12.6 \%(233 / 1769)$ of hospital patients with no observable PUs reported pressure area related pain. In addition, substudies involving patients who consented to a detailed pain assessment, 157 hospital and 37 community patients reported pain on $68.0 \%$ (66/97) and 19/20 (95\%) skin sites assessed as grade 1 (ie, intact skin with non-blanching erythema).$^{78}$

The results indicated the extent of pressure area related pain in hospital inpatients with skin assessed as 'normal' or 'non-blanching but intact'.

In the second stage of the programme, we explored the role of pressure area related pain of intact skin as a predictor of PU development in acute hospital and community populations.

\section{Aims and objectives}

The primary aim was to explore the role of pressure area related pain as an early predictor of category $\geq 2$ PU development in patients at high risk of PU development. Objectives were to:

1. Assess whether the presence/absence of pressure area related pain is a predictor of category $\geq 2 \mathrm{PU}$ development.

2. Identify variables which are independently predictive of category $\geq 2$ PU development.

\section{METHODS}

\section{Study design}

We conducted a prospective cohort study involving acutely ill hospital and community patients at high risk of PU development. Follow-up was twice weekly for a maximum of 30 days from registration or until they were no longer at high risk of PU development or transferred to a non-participating centre or death.

\section{Setting}

Patients were recruited from 26 hospital and community centres across 18 NHS Trusts in England between 26 October 2009 and 17 November 2011. All centres had PU prevention and management policies and guidelines based on national and international guidelines ${ }^{9}$ including risk assessment and mattress provision and patients received care as determined by the attending clinical teams.

\section{Patients}

All patients were aged $\geq 18$ years, provided written informed consent, were able to report the presence/ absence of pressure area related pain, were at high risk of PU development and indicated as acutely ill.
High risk of PU development was defined as one or more of the following: (1) bedfast/chairfast and completely immobile/have very limited mobility according to the Braden Scale, ${ }^{10}$ (2) localised skin pain on any pressure area skin site and (3) category 1 PU on any pressure area skin site. ${ }^{2411}$

Indicators of acute illness were defined pragmatically as one of the following: acute hospital admission to vascular, orthopaedic, medical or care of the elderly specialties $;{ }^{2}{ }^{11}$ new community nursing referral following hospital discharge to home/intermediate/community care/hospice/specialist palliative care; existing community nursing patient with deterioration in overall condition or onset of acute illness; new referral to community nursing due to acute illness, deterioration in existing condition or care package breakdown.

Patients were not eligible to participate if they were admitted to obstetrics, paediatrics, day case surgery or psychiatric wards. Patients were also not eligible if they were deemed by the attending healthcare professional to be too unwell to be approached and/or complete the study assessment schedule, or they had $\geq 2$ existing category $\geq 2$ PUs on the following pressure area skin sites: sacrum, buttocks, heels and hips.

Eligible patients were recruited by the research team and after written informed consent/witnessed verbal consent they were registered to the study using a central 24-hour telephone registration system.

\section{Outcomes}

The primary outcome was the development of a new category $\geq 2$ PU. This was defined on a skin site level basis before being combined into a patient level outcome (figure 1). Secondary outcomes were time to development of the first PU and development of a category $\geq 2$ $\mathrm{PU}$ at the skin site level.

\section{Data sources/measurement}

Baseline assessment was conducted by trained clinical research nurses and included: demographics (age, gender, setting); a priori risk factors (diabetic status, history of prior weight loss, body mass index (BMI), mattress category, analgesic use, and Braden subdomains) ${ }^{2} 10$ and skin and pain status.

Skin assessment was conducted on 13 prespecified pressure area skin sites (left and right buttocks, heels, elbows, ankles, trochanters, ischial tuberosities and sacrum) and any additional pressure areas identified by the research nurse doing the assessment using the NPUAP/EPUAP PU classification. ${ }^{9}$ PUs were classified as category 1-4 or unstageable. In addition, as general skin condition is predictive of category $\geq 2$ PU development, ${ }^{2} 1213$ observations of any alteration to intact skin (eg, redness, scar, excoriation, dry, scaly) were recorded and the presence of healthy skin or other wounds was confirmed for each skin site.

Pressure area related pain was defined as pain, soreness or discomfort on any intact pressure area (ie, skin 
Figure 1 Derivation of skin site level and patient level outcomes. *Evaluable skin sites are such that skin site level outcome can be determined as 'Develops PU' or 'Does not develop PU'. Note that patients had up to nine assessments in total (baseline and twice weekly for up to 30 days). PU, pressure ulcer.

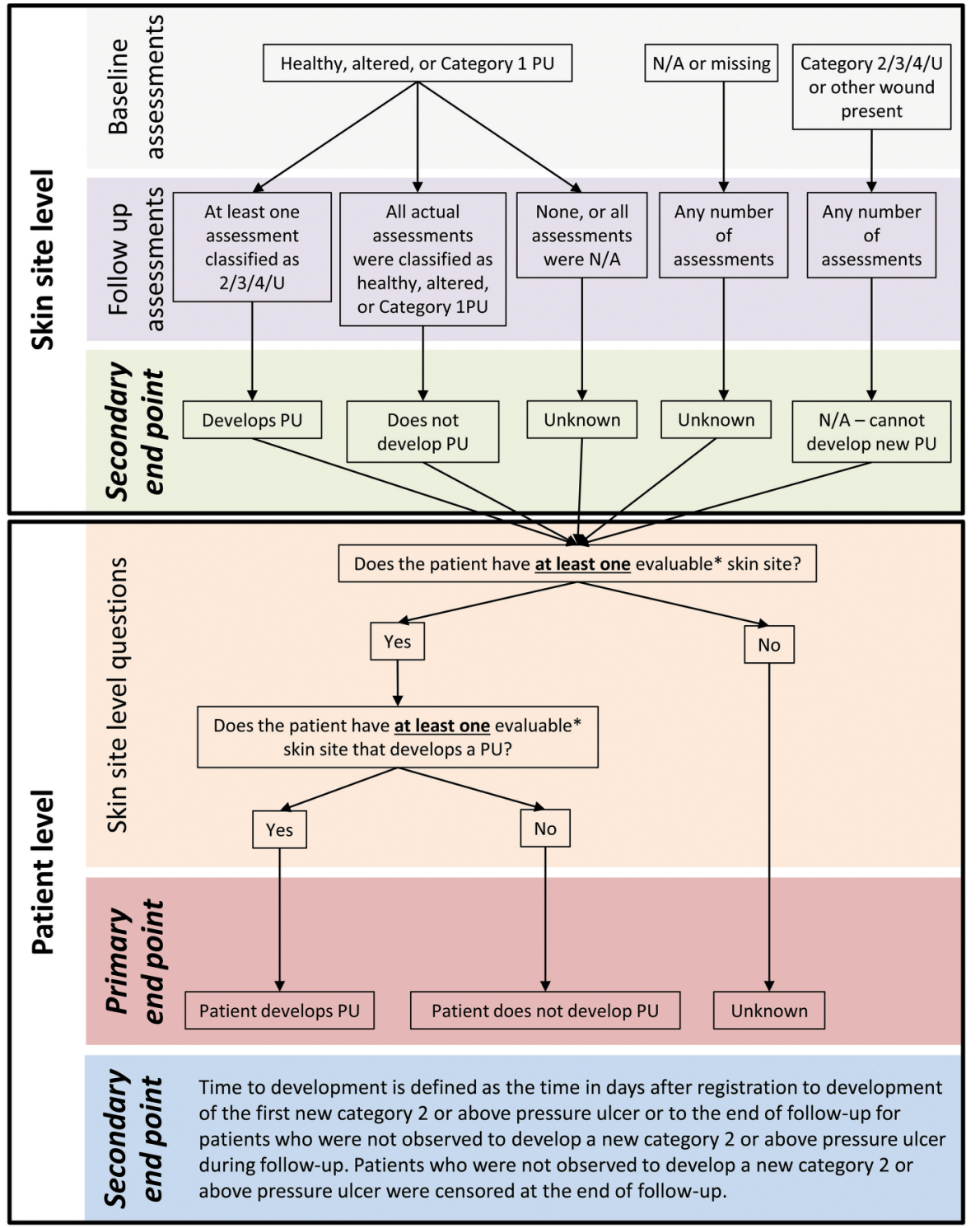

status assessed as normal, altered or category 1). ${ }^{7}$ To determine if patients had localised skin pain on any pressure area skin site, they were asked two screening questions with yes or no responses: (1) at any time, do you get pain, soreness or discomfort at a pressure area (prompt: back, bottom, heels, elbows or other as appropriate to the patient)? and (2) do you think this is related to either; your PU OR lying in bed for a long time OR sitting for a long time? ${ }^{7} 8$

Patients were followed up twice weekly for 30 days postregistration or until one of the following criteria applied: (1) no longer at high risk of PU development, (2) transferred to non-participating centre and (3) death.

\section{Bias}

Anonymised screening logs were maintained by centres, and these were monitored to ensure that centres were approaching and recruiting appropriate patients. While blinded end point assessment was not possible, the risk factor and outcome data were recorded by research staff independent of the clinical team.

\section{Sample size}

For risk factor studies using logistic regression, it is recommended that at least 10 patients with the event of interest are required for reliable estimation of effects. ${ }^{14}$ Our primary model planned to include a maximum of 9 factors and therefore required at least 90 patients to develop a new category $\geq 2$ PU. In the absence of prospective data for community-based patient populations, ${ }^{2}$ the sample size estimate was based on previous research in hospital patients, ${ }^{11} 13$ suggesting that $\sim 15 \%$ of patients would develop a new category $\geq 2$ PU within 30 days of registration. Therefore, assuming loss to follow-up of $5 \%$, the study required 632 patients.

A further consideration in appraising the sample size estimate was the prevalence of pain at study entry. As no previous work in this field had been undertaken, we considered a range of prevalence rates. We estimated that if we recruited 600 patients (after accounting for $5 \%$ loss to follow-up) with $60(10 \%)$ patients having pressure-related pain at baseline, this would allow us to detect a difference of $13.2 \%$ between those with and 
those without pain using a $\chi^{2}$ test $(80 \%$ power, two-sided $5 \%$ significance level) assuming $10 \%$ of patients without pain and $23.2 \%$ of those with pain developed a new PU within 30 days, corresponding to an OR of 2.72 with $95 \%$ CI of (1.40 to 5.27). As this was an exploratory study and there was uncertainty around the sample size assumptions, the proportion of patients with pain at baseline and PU incidence was monitored by the statistical team and chief investigator and reported to a subgroup of the Programme Steering Committee.

\section{Statistical methods}

All statistical analyses were carried out in SAS V.9.2 and based on the evaluable patient population. That is, all patients for whom at least one skin site was evaluable (figure 1).

\section{Primary analysis: logistic regression model}

Univariable logistic regression was conducted to assess a priori variables for inclusion in the multivariable logistic regression model. The a priori variables included the presence of pressure area related pain on a healthy, altered skin or category 1 skin site and other risk factors based on a conceptual framework: ${ }^{16}$ age, diabetes, history of prior weight loss, Braden mobility subscale (completely immobile/very limited mobility vs slightly limited mobility/no limitation with mobility), presence of skin alterations, presence of a category $1 \mathrm{PU}$ and setting (hospital vs community). Candidate variables were considered to be statistically significant and therefore associated with the development of a category $\geq 2$ PU if the $p$ value was $<0.1$ for the associated likelihood ratio test (LRT). Full stepwise variable selection was conducted to build a multivariable logistic regression model for the odds of developing a category $\geq 2$ PU. Candidate variables were retained in the model if their exclusion led to an increase in deviance with a corresponding $\mathrm{p}$ value that was $>0.1$ for the associated LRT. ORs, corresponding 95\% CIs and $p$ values are presented for the univariable and final multivariable models. The level of 0.1 was chosen in line with recommendations for model selection in the analysis of binary data. ${ }^{17}$

Three secondary analyses were conducted as follows.

Overdispersion model: Overdispersion model was undertaken to determine if the model could be improved by inclusion of other variables. Further baseline variables (gender, BMI, Braden Scale domains (activity, friction and shear, moisture, nutrition and sensory perception), presence of a category $\geq 2 \mathrm{PU}$, presence of chronic wound and type of mattress) ${ }^{2}{ }^{16}$ were considered for inclusion in the final model from the primary analysis using full stepwise variable selection and the same criteria as for the primary analysis. ORs, corresponding 95\% CIs and $\mathrm{p}$ values are presented for the additional univariable and final multivariable models.

Accelerated failure time model: Full stepwise variable selection was considered for each of the following distributions: Gamma, log-logistic, log-normal and exponential. The model fit of the final models obtained for each distribution were explored using Cox-Snell residual plots. The Gamma distribution was observed to be the most appropriate and so, an accelerated failure time (AFT) model assuming the Gamma distribution was fitted to the data to explore the relationship between the presence of pressure area related pain and time to onset of the first new category $\geq 2 \mathrm{PU}$. Univariable analyses were conducted to determine which variables were associated with time to onset of a new category $\geq 2 \mathrm{PU}$, and the final AFT model was obtained using full stepwise variable selection with the same criteria as for the primary analysis. Acceleration factors, corresponding 95\% CIs and $p$ values are presented for the additional univariable and final multivariable models.

Skin site level model: Nesting of skin sites within patients was taken into account using a two-level random-intercept logistic regression model. Univariable analyses and full stepwise variable selection were conducted using the same selection criteria as for the primary analysis to obtain the final multivariable model. For this multilevel model, only evaluable skin sites (figure 1) were analysed, and the variables considered for inclusion in the model were modified to reflect the structure of the data such that age, diabetic status, history of prior weight loss, Braden mobility, setting and analgesic use continued to be patient level risk factors. The presence of pressure area related pain at baseline was assessed for each skin site and was therefore included as a skin site level risk factor, and the presence of skin alterations and the presence of a category $1 \mathrm{PU}$ were combined into a single skin site level risk factor (healthy/skin alterations/category $1 \mathrm{PU}$ ). ORs, corresponding 95\% CIs and $\mathrm{p}$ values are presented for the univariable and final multivariable models.

\section{Quantitative variables}

Age was considered twice for inclusion in the model as a categorical variable ( $<65$ years $/ 65-74$ years $/ 75-84$ years/ $\geq 85$ years) and as a continuous variable. The Braden mobility subscale was dichotomised (completely immobile/very limited mobility vs slightly limited mobility/no limitation with mobility), and all remaining variables for inclusion in the primary model were considered to be binary variables. For the secondary analysis model, BMI was considered for inclusion as a continuous variable, the remaining Braden subscales were considered on an ordinal scale, mattress type was categorised as dynamic high-risk pressure-relieving/static high-risk pressurerelieving/non-pressure relieving and the remaining variables for inclusion in the secondary analysis model for the primary outcome were considered to be binary variables.

\section{RESULTS}

\section{Patients}

A total of 3819 patients were screened for participation in the study. A total of 1266 were eligible and of these 
634 were registered between 26 October 2009 and 17 November 2011. Reasons for ineligibility and refusals/ non-registration are detailed in figure 2. Thirty-two (5.0\%) patients did not have any follow-up assessments, and the analysis population therefore comprised 602 evaluable patients (figure 2), including 397 (65.9\%) hospital and $205(34.1 \%)$ community evaluable patients.
Baseline characteristics are detailed in table 1 . The median (range) length of follow-up was 27 (1-34) days.

\section{Descriptive data}

A total of $152(25.2 \%)$ patients developed 223 new category $\geq 2$ PUs during follow-up. There were $464(77.1 \%)$ patients with pressure area related pain on at least one

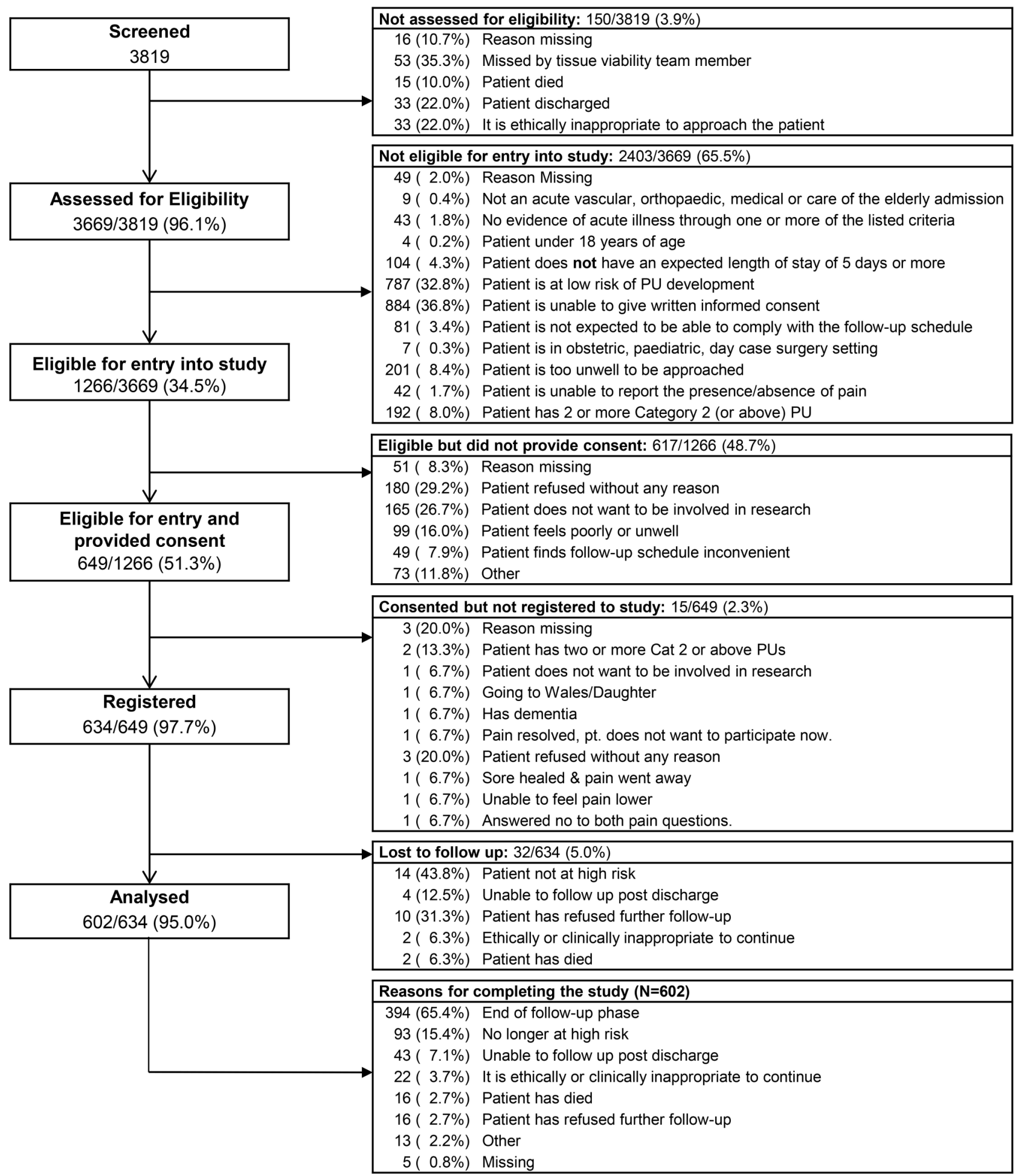

Figure 2 Patient flow. 
Table 1 Patient level baseline characteristics by setting

\begin{tabular}{|c|c|c|c|}
\hline Variable & Hospital $(\mathrm{N}=397)$ & Community (N=205) & Total $(\mathrm{N}=602)$ \\
\hline \multicolumn{4}{|l|}{ Specialty or place assessed } \\
\hline Vascular & $42(10.6 \%)$ & $0(0.0 \%)$ & $42(7.0 \%)$ \\
\hline Orthopaedic & $155(39.0 \%)$ & $0(0.0 \%)$ & $155(25.7 \%)$ \\
\hline Medical & $90(22.7 \%)$ & $0(0.0 \%)$ & $90(15.0 \%)$ \\
\hline Elderly & $32(8.1 \%)$ & $0(0.0 \%)$ & $32(5.3 \%)$ \\
\hline Medical/elderly & $78(19.6 \%)$ & $0(0.0 \%)$ & $78(13.0 \%)$ \\
\hline Patient's own home & $0(0.0 \%)$ & $49(23.9 \%)$ & $49(8.1 \%)$ \\
\hline Nursing home & $0(0.0 \%)$ & $27(13.2 \%)$ & 27 (4.5\%) \\
\hline Residential home & $0(0.0 \%)$ & $18(8.8 \%)$ & $18(3.0 \%)$ \\
\hline Rehabilitation unit & $0(0.0 \%)$ & $104(50.7 \%)$ & $104(17.3 \%)$ \\
\hline Other place assessed & $0(0.0 \%)$ & $7(3.4 \%)$ & $7(1.2 \%)$ \\
\hline \multicolumn{4}{|l|}{ Age } \\
\hline Mean (SD) & $75.6(12.9)$ & $80.7(11.7)$ & $77.3(12.7)$ \\
\hline Median (range) & $79(21,101)$ & $83(30,100)$ & $80(21,101)$ \\
\hline Male & $156(39.3 \%)$ & $77(37.6 \%)$ & $233(38.7 \%)$ \\
\hline Caucasian & $396(99.7 \%)$ & 205 (100.0\%) & $601(99.8 \%)$ \\
\hline Diabetic & $98(24.7 \%)$ & $55(26.8 \%)$ & $153(25.4 \%)$ \\
\hline $\mathrm{N}$ missing & $0(0.0 \%)$ & $1(0.5 \%)$ & $1(0.2 \%)$ \\
\hline History of prior weight loss & $95(23.9 \%)$ & $52(25.4 \%)$ & $147(24.4 \%)$ \\
\hline $\mathrm{N}$ missing & $0(0.0 \%)$ & $1(0.5 \%)$ & $1(0.2 \%)$ \\
\hline \multicolumn{4}{|l|}{ BMI } \\
\hline Mean (SD) & $27.1(9.3)$ & $26.0(9.9)$ & $26.7(9.5)$ \\
\hline Median (range) & $25(11,94)$ & $24(11,111)$ & $25(11,111)$ \\
\hline $\mathrm{N}$ missing & $12(3.0 \%)$ & $12(5.9 \%)$ & $24(4.0 \%)$ \\
\hline Chronic wound at baseline & $75(18.9 \%)$ & $52(25.4 \%)$ & $127(21.1 \%)$ \\
\hline $\mathrm{N}$ missing & $0(0.0 \%)$ & $1(0.5 \%)$ & $1(0.2 \%)$ \\
\hline \multicolumn{4}{|l|}{ Worst skin status at baseline } \\
\hline Healthy & $45(11.3 \%)$ & $25(12.2 \%)$ & $70(11.6 \%)$ \\
\hline Alterations to intact skin & $99(24.9 \%)$ & $55(26.8 \%)$ & $154(25.6 \%)$ \\
\hline Category 1 & $137(34.5 \%)$ & $77(37.6 \%)$ & $214(35.5 \%)$ \\
\hline Category 2 & $98(24.7 \%)$ & $42(20.5 \%)$ & $140(23.3 \%)$ \\
\hline Category 3 & $10(2.5 \%)$ & $3(1.5 \%)$ & $13(2.2 \%)$ \\
\hline Category 4 & $2(0.5 \%)$ & $2(1.0 \%)$ & $4(0.7 \%)$ \\
\hline Unstageable & $6(1.5 \%)$ & $1(0.5 \%)$ & $7(1.2 \%)$ \\
\hline Analgesic in use & $366(92.2 \%)$ & $182(88.8 \%)$ & $548(91.0 \%)$ \\
\hline $\mathrm{N}$ missing & $0(0.0 \%)$ & $0(0.0 \%)$ & $0(0.0 \%)$ \\
\hline \multicolumn{4}{|l|}{ Braden mobility } \\
\hline Completely immobile & $9(2.3 \%)$ & $12(5.9 \%)$ & $21(3.5 \%)$ \\
\hline Very limited & $204(51.4 \%)$ & $58(58.3 \%)$ & $262(43.5 \%)$ \\
\hline Slightly limited & $142(35.8 \%)$ & 89 (43.4\%) & $231(38.4 \%)$ \\
\hline No limitation & $42(10.6 \%)$ & $46(22.4 \%)$ & $88(14.6 \%)$ \\
\hline \multicolumn{4}{|l|}{ Mattress type } \\
\hline Non-pressure relieving & $5(1.3 \%)$ & $31(15.1 \%)$ & $36(6.0 \%)$ \\
\hline Static pressure-relieving & $168(42.3 \%)$ & $105(51.2 \%)$ & $273(45.3 \%)$ \\
\hline Dynamic pressure-relieving & $224(56.4 \%)$ & $68(33.2 \%)$ & $292(48.5 \%)$ \\
\hline Missing & $0(0.0 \%)$ & $1(0.5 \%)$ & $1(0.2 \%)$ \\
\hline
\end{tabular}

healthy, altered or category 1 skin site at baseline; $28.0 \%$ $(\mathrm{N}=130)$ of these developed a new category $\geq 2$ PU compared to $15.9 \%(\mathrm{~N}=22)$ of those without pain at baseline (table 2). The summary of worst skin status indicates that a total of $532(88.4 \%)$ patients had some skin damage at baseline.

The analysis population of 602 patients had a combined total of 7863 potential skin sites assessed corresponding to 13 prespecified skin sites for each of the 602 patients and 37 additional 'other' skin sites. The majority $(77.5 \%)$ of skin sites were observed as being healthy skin sites, and pain was reported more frequently with severity of skin status (table 3); for example, $63.1 \%(351 / 556)$ of category 1 skin sites were observed to have pain compared to $40.3 \%$ (342/849) of skin sites with alterations and $6.4 \%(390 / 6096)$ of healthy skin sites. After excluding 172 skin sites with an existing category $\geq 2 \mathrm{PU}, 183$ skin sites with no baseline assessment (eg, amputation or bandage in situ), 7 assessed as another wound and 18 with no follow-up 
Table 2 Development of PU on a patient level by skin and pain status

\begin{tabular}{|c|c|c|c|}
\hline Variable & $\begin{array}{l}\text { Develops } \\
\text { new PU } \\
(\mathrm{N}=152)\end{array}$ & $\begin{array}{l}\text { Does not } \\
\text { develop new } \\
\text { PU }(\mathrm{N}=450)\end{array}$ & Total $(\mathrm{N}=602)$ \\
\hline \multicolumn{4}{|c|}{ Presence of skin alterations at baseline } \\
\hline Yes & $109(29.2 \%)$ & $264(70.8 \%)$ & $373(62.0 \%)$ \\
\hline No & $43(18.8 \%)$ & $186(81.2 \%)$ & $229(38.0 \%)$ \\
\hline \multicolumn{4}{|c|}{ Presence of category $1 \mathrm{PU}$ at baseline } \\
\hline Yes & $105(36.2 \%)$ & $185(63.8 \%)$ & $290(48.2 \%)$ \\
\hline No & $47(15.1 \%)$ & $265(84.9 \%)$ & $312(51.8 \%)$ \\
\hline \multicolumn{4}{|c|}{$\begin{array}{l}\text { Presence of pain at a healthy, altered or category } 1 \mathrm{PU} \\
\text { skin site }\end{array}$} \\
\hline Yes & $130(28.0 \%)$ & $334(72.0 \%)$ & $464(77.1 \%)$ \\
\hline No & $22(15.9 \%)$ & $116(84.1 \%)$ & $138(22.9 \%)$ \\
\hline
\end{tabular}

assessment, $7483(95.2 \%)$ skin sites were evaluable in terms of obtaining a skin site level outcome and were therefore considered for the skin site level analysis.

Of the evaluable skin sites, a total of $223(3.0 \%)$ developed a new category $\geq 2$ PU. The proportion of skin sites developing a new category $\geq 2$ PU appears to increase with severity of baseline skin status with $1.1 \%$ of healthy skin sites, $6.4 \%$ of altered skin sites and $18.2 \%$ of category 1 PU skin sites observed to develop new category $\geq 2$ PUs (table 4). There were 1077 (14.4\%) skin sites with pressure area related pain at baseline and $10.3 \%$ of these developed a new category $\geq 2$ PU compared to $1.7 \%$ of the skin sites without pressure-related pain (table 4 ).

\section{Logistic regression model}

In the univariable analysis, diabetic status, presence of skin alterations, presence of category 1 and pressure area related pain were statistically significantly associated with the odds of developing a new category $\geq 2$ PU (table 5). Following full stepwise variable selection, the
Table 4 Development of category $\geq 2$ PU by pain and skin assessments on a skin site level basis

\begin{tabular}{|c|c|c|c|}
\hline Variable & $\begin{array}{l}\text { Develops } \\
\text { new PU } \\
\text { (N=223) }\end{array}$ & $\begin{array}{l}\text { Does not } \\
\text { develop } \\
\text { new PU } \\
(\mathrm{N}=7260)\end{array}$ & $\begin{array}{l}\text { Total } \\
\left(\mathrm{N}=7483^{\star}\right)\end{array}$ \\
\hline \multicolumn{4}{|l|}{ Pain } \\
\hline Pain yes & $111(10.3 \%)$ & $966(89.7 \%)$ & 1077 (14.4\%) \\
\hline Pain no & $112(1.7 \%)$ & $6294(98.3 \%)$ & $6406(85.6 \%)$ \\
\hline \multicolumn{4}{|l|}{ Skin status } \\
\hline $\begin{array}{l}\text { Healthy } \\
\text { skin }\end{array}$ & $68(1.1 \%)$ & $6014(98.9 \%)$ & $6082(81.3 \%)$ \\
\hline $\begin{array}{l}\text { Alterations } \\
\text { to intact } \\
\text { skin }\end{array}$ & $54(6.4 \%)$ & 792 (93.6\%) & $846(11.3 \%)$ \\
\hline Category 1 & $101(18.2 \%)$ & 454 (81.8\%) & $555(7.4 \%)$ \\
\hline
\end{tabular}

${ }^{*}$ Note that 172 skin sites with an existing Category $\geq 2$ PU, 183 skin sites with no baseline assessment (eg, amputation or bandage in situ) and 25 with no follow-up assessment were excluded leaving a total of 7483 skin sites for analysis.

PU, pressure ulcer.

final model (602 patients) indicates that the odds of developing a category $\geq 2$ PU were increased for the following variables: category 1 PU (OR 3.25(95\% CI (2.17 to 4.86$)$ ), $\mathrm{p}<0.0001)$, presence of skin alterations (OR $(95 \% \mathrm{CI})=1.98(1.30$ to 3.00$), \mathrm{p}=0.0014)$ and presence of pressure area related pain (OR $(95 \% \mathrm{CI})=1.56$ (0.93 to 2.63), $\mathrm{p}=0.0931)$ (table 5).

\section{Overdispersion analysis}

Univariable analyses of additional variables indicated that gender, Braden moisture, Braden activity, presence of a chronic wound and presence of pre-existing category 2 PU were statistically significantly associated with the odds of developing a new category $\geq 2 \mathrm{PU}$ (table 6 ).

The final overdispersion model retained the variables (category $1 \mathrm{PU}$, skin alterations and the presence of pressure-related pain) from the final primary analysis

Table 3 Baseline skin and pain assessment on a skin site level basis

\begin{tabular}{|c|c|c|c|c|}
\hline Skin classification & $\begin{array}{l}\text { Pain yes } \\
\text { N (\%) }\end{array}$ & $\begin{array}{l}\text { Pain no } \\
\text { N (\%) }\end{array}$ & $\begin{array}{l}\text { Missing } \\
\text { N (\%) }\end{array}$ & $\begin{array}{l}\text { Total } \\
\text { N (\%) }\end{array}$ \\
\hline Healthy skin & $390(6.4 \%)$ & 5700 (93.5\%) & $6(0.1 \%)$ & $6096(77.5 \%)$ \\
\hline Skin alterations & $342(40.3 \%)$ & $504(59.4 \%)$ & $3(0.4 \%)$ & $849(10.8 \%)$ \\
\hline Category 1 & $351(63.1 \%)$ & $205(36.9 \%)$ & $0(0.0 \%)$ & $556(7.1 \%)$ \\
\hline Category 2 & $116(78.4 \%)$ & $32(21.6 \%)$ & $0(0.0 \%)$ & 148 (1.9\%) \\
\hline Category 3 & $13(100 \%)$ & $0(0.0 \%)$ & $0(0.0 \%)$ & $13(0.2 \%)$ \\
\hline Category 4 & $3(75.0 \%)$ & $1(25.0 \%)$ & $0(0.0 \%)$ & $4(0.1 \%)$ \\
\hline Unstageable & $6(85.7 \%)$ & 1 (14.3\%) & $0(0.0 \%)$ & $7(0.1 \%)$ \\
\hline Unable to assess & $6(7.6 \%)$ & 52 (65.8\%) & $21(26.6 \%)$ & 79 (1.0\%) \\
\hline Not applicable & $2(2.3 \%)$ & $26(29.9 \%)$ & $59(67.8 \%)$ & $87(1.1 \%)$ \\
\hline Other wound & $0(0.0 \%)$ & $6(85.7 \%)$ & $1(14.3 \%)$ & $7(0.1 \%)$ \\
\hline Classification missing & $0(0.0 \%)$ & $0(0.0 \%)$ & $17(100 \%)$ & $17(0.2 \%)$ \\
\hline Total & $1229(15.6 \%)$ & $6527(83.0 \%)$ & $107(1.4 \%)$ & $7863(100.0 \%)$ \\
\hline
\end{tabular}

NB: 602 patients. Each patient had 13 skin assessments and there were 37 'other' sites assessed. The overall total therefore corresponds to $(602 \times 13)+37=7863$ skin assessments. 
Table 5 Primary analysis (patient level): logistic regression models

\begin{tabular}{|c|c|c|c|}
\hline Covariate & OR & $95 \% \mathrm{Cl}$ & p Value \\
\hline \multicolumn{4}{|l|}{ Univariable analyses } \\
\hline Age (continuous) & 1.01 & 0.99 to 1.022 & 0.3936 \\
\hline \multicolumn{4}{|l|}{ Age (categorical) (reference='less than 65 years') } \\
\hline 85 years or older & 1.31 & 0.73 to 2.35 & 0.4192 \\
\hline $65-74$ years & 1.20 & 0.63 to 2.29 & 0.3885 \\
\hline $75-84$ years & 1.17 & 0.65 to 2.08 & 0.2996 \\
\hline Diabetic status* (yes vs no) & 1.45 & 0.97 to 2.18 & 0.0722 \\
\hline History of weight loss ${ }^{\dagger}$ (yes vs no) & 1.04 & 0.68 to 1.60 & 0.8462 \\
\hline Braden mobility ( 1 or 2 vs 3 or 4 ) & 1.21 & 0.84 to 1.76 & 0.3055 \\
\hline Skin alterations (yes vs no) & 1.79 & 1.20 to 2.66 & 0.0045 \\
\hline Category $1 \mathrm{PU}$ (yes vs no) & 3.20 & 2.63 to 4.74 & $<0.0001$ \\
\hline Setting (hospital vs community) & 0.92 & 0.62 to 1.35 & 0.6576 \\
\hline Analgesic use (yes vs no) & 0.87 & 0.46 to 1.62 & 0.6542 \\
\hline $\begin{array}{l}\text { Pressure-related pain on healthy, altered } \\
\text { or category } 1 \text { skin site (yes vs no) }\end{array}$ & 2.05 & 1.25 to 3.38 & 0.0047 \\
\hline \multicolumn{4}{|l|}{ Final multivariable model from primary analysis } \\
\hline Category $1 \mathrm{PU}$ (yes vs no) & 3.25 & 2.17 to 4.86 & $<0.0001$ \\
\hline Skin alterations (yes vs no) & 1.98 & 1.30 to 3.00 & 0.0014 \\
\hline $\begin{array}{l}\text { Pressure-related pain on healthy, altered or Category } 1 \\
\text { skin site (yes vs no) }\end{array}$ & 1.56 & 0.93 to 2.63 & 0.0931 \\
\hline
\end{tabular}

model and the presence of pre-existing category $2 \mathrm{PU}$ $(\mathrm{OR}(95 \% \mathrm{CI})=2.09$ (1.35 to 3.23), $\mathrm{p}=0.0009)$, presence of chronic wound (OR $(95 \% \mathrm{CI})=1.66$ (1.06 to 2.62), $\mathrm{p}=0.0277$ ) and Braden Activity subscale ( $\mathrm{p}$ value for overall analysis of effects $=0.0476$ ). Within this model, the estimate of the odds of category $\geq 2$ PU development in the presence of pressure area related pain increased $(\mathrm{OR}(95 \% \mathrm{CI})=1.85$ (1.07 to 3.20$), \mathrm{p}=0.0271)$ (table 6$)$.

\section{Accelerated failure time model}

The univariable AFT analyses indicated that age, presence of skin alterations, Braden mobility, presence of category $1 \mathrm{PU}$ and presence of pressure area related pain were statistically significantly associated with increased rate of category $\geq 2 \mathrm{PU}$ development (table 7 ).

The final AFT model included presence of a category 1 PU (AF $(95 \% \mathrm{CI})=2.32(1.73$ to 3.12$), \mathrm{p}<0.0001)$ and presence of pressure area related pain $(\mathrm{AF}(95 \% \mathrm{CI})=2.28(1.59$ to 3.27$), \mathrm{p}<0.0001)$. The model indicates that patients are likely to develop a category $\geq 2$ PU 2.32 times faster compared to patients who do not have a category $1 \mathrm{PU}$ (AF $(95 \% \mathrm{CI})=2.32(1.73$ to 3.12$))$. In addition, patients with pressure area related pain are likely to develop a category $\geq 2$ PU 2.28 times faster (95\% CI (1.59 to 3.27)) compared to patients who do not have pressure-related pain.

\section{Skin site level model}

The univariable analyses indicated that skin status and the presence of pain were statistically significantly associated with the odds of developing a category $\geq 2 \mathrm{PU}$ at the same skin site (table 8 ).
The final multivariable multilevel logistic regression model obtained after full stepwise variable selection indicates that skin status and the presence of pressure-related pain are predictive of PU development at the same skin site, after adjusting for patient variation, that is the clustering of skin sites within patients: presence of skin alterations (OR $(95 \% \mathrm{CI})=4.65$ ((3.01 to 7.18), $\mathrm{p}<0.0001$, compared to healthy skin), presence of a category $1 \mathrm{PU}(\mathrm{OR}(95 \% \mathrm{CI})=17.30 \quad(11.09$ to 27.00$)$, $\mathrm{p}<0.0001$, compared to healthy skin) and presence of pressure area related pain $(\mathrm{OR}(95 \% \mathrm{CI})=2.25$ (1.53 to 3.29), $\mathrm{p}<0.0001)$.

\section{DISCUSSION}

\section{Statement of principal findings}

This is the first risk factor study to investigate pain as a risk factor for PU development and found that pain was an independent predictor of category $\geq 2$ PU development in high risk hospital and community patients with acute illness. There was significant evidence that the presence of pressure area related pain is an independent predictor for developing a category $\geq 2 \mathrm{PU}$, after adjusting for skin status at baseline, across all four multivariable models. On a patient level, there was marginal evidence that the presence of pain increased the odds for developing a category $\geq 2$ PU by 30 days of follow-up (primary end point), and significant evidence that the presence of pressure area related pain also accelerates the time to developing a category $\geq 2 \mathrm{PU}$, after adjusting for other important covariates. On a skin site level, 
Table 6 Overdispersion analysis (patient level): logistic regression models

\begin{tabular}{|c|c|c|c|}
\hline Covariate & OR & $95 \% \mathrm{Cl}$ & p Value \\
\hline \multicolumn{4}{|l|}{ Univariable analyses } \\
\hline Gender (female vs male) & 0.72 & 0.49 to 1.04 & 0.0780 \\
\hline BMI (continuous) & 1.00 & 0.99 to 1.02 & 0.6702 \\
\hline \multicolumn{4}{|l|}{ Braden sensory (reference=No impairment) } \\
\hline Slightly Limited & 0.92 & 0.54 to 1.58 & \multirow[t]{2}{*}{0.6886} \\
\hline Very Limited & 0.41 & 0.05 to 3.40 & \\
\hline \multicolumn{4}{|l|}{ Braden Moisture (reference=Rarely moist) } \\
\hline Occasionally moist & 1.83 & 1.22 to 2.74 & \multirow[t]{3}{*}{0.0277} \\
\hline Very moist & 1.11 & 0.51 to 2.44 & \\
\hline Constantly moist & 2.39 & 0.39 to 14.55 & \\
\hline \multicolumn{4}{|l|}{ Braden activity (reference=Bedfast) } \\
\hline Chairfast & 1.83 & 1.05 to 3.19 & \multirow[t]{3}{*}{0.0775} \\
\hline Walks occasionally & 1.43 & 0.77 to 2.65 & \\
\hline Walks frequently & 0.75 & 0.23 to 2.40 & \\
\hline \multicolumn{4}{|l|}{ Braden nutrition (reference=Excellent) } \\
\hline Adequate & 0.93 & 0.59 to 1.47 & \multirow[t]{3}{*}{0.6058} \\
\hline Probably inadequate & 1.26 & 0.77 to 2.06 & \\
\hline Very poor & 1.10 & 0.37 to 3.23 & \\
\hline \multicolumn{4}{|l|}{ Braden friction and shear (reference $=$ No apparent problem) } \\
\hline Potential problem & 1.06 & 0.62 to 1.82 & \multirow[t]{2}{*}{0.8036} \\
\hline Problem & 1.22 & 0.64 to 2.36 & \\
\hline \multicolumn{4}{|l|}{ Mattress category (reference $=$ dynamic high risk pressure relieving } \\
\hline Static risk pressure relieving & 1.28 & 0.87 to 1.87 & \multirow[t]{2}{*}{0.2430} \\
\hline Non-pressure relieving & 0.95 & 0.41 to 2.17 & \\
\hline Chronic wound (yes vs no) & 1.89 & 1.24 to 2.88 & 0.0032 \\
\hline Category $\geq 2 \mathrm{PU}$ (yes vs no) & 1.70 & 1.15 to 2.53 & 0.0083 \\
\hline \multicolumn{4}{|l|}{ Final multivariable model from secondary overdispersion analysis } \\
\hline Category $1 \mathrm{PU}$ (yes vs no) & 3.20 & 2.11 to 4.85 & $<0.0001$ \\
\hline Skin alterations (yes vs no) & 1.90 & 1.24 to 2.91 & 0.0032 \\
\hline Pressure-related pain on healthy, altered or category 1 skin site (yes vs no) & 1.85 & 1.07 to 3.20 & 0.0271 \\
\hline Category 2 PU (yes vs no) & 2.09 & 1.35 to 3.23 & 0.0009 \\
\hline Braden activity: Chairfast vs Bedfast & 1.86 & 1.03 to 3.36 & 0.0476 \\
\hline Braden activity: Walks occasionally vs Bedfast & 1.19 & 0.62 to 2.29 & \\
\hline Braden Activity: Walks frequently $v$ Bedfast & 0.71 & 0.21 to 2.46 & \\
\hline Chronic wound (yes vs no) & 1.66 & 1.06 to 2.62 & 0.0277 \\
\hline
\end{tabular}

Bold values indicate statistical significance.

$\mathrm{BMI}$, body mass index; PU, pressure ulcer.

Table 7 Analysis of time to PU development (patient level): Accelerated Failure Time models

\begin{tabular}{|c|c|c|c|}
\hline Covariate & $\begin{array}{l}\text { Ratio* of time to developing } \\
\text { new Category } \geq 2 \mathrm{PU}\end{array}$ & $95 \% \mathrm{Cl}$ & p Value \\
\hline \multicolumn{4}{|l|}{ Univariable analyses } \\
\hline Age (continuous) & 1.01 & 1.00 to 1.03 & 0.0354 \\
\hline Skin alterations (yes vs no) & 1.40 & 0.99 to 1.97 & 0.0593 \\
\hline Analgesic use (yes vs no) & 1.05 & 0.61 to 1.83 & 0.8577 \\
\hline Braden mobility ( 1 or 2 vs 3 or 4 ) & 1.37 & 1.00 to 1.87 & 0.0498 \\
\hline Category 1 PU (yes vs no) & 2.63 & 1.93 to 3.58 & $<0.0001$ \\
\hline Diabetic (yes vs no) & 1.24 & 0.85 to 1.80 & 0.2591 \\
\hline History of prior weight loss (yes vs no) & 0.90 & 0.62 to 1.30 & 0.5715 \\
\hline Pain (yes vs no) & 2.68 & 1.86 to 3.86 & $<0.0001$ \\
\hline Setting (Hospital vs community) & 1.13 & 0.81 to 1.58 & 0.4652 \\
\hline \multicolumn{4}{|l|}{ Final multivariable AFT model } \\
\hline Category 1 PU (yes vs no) & 2.32 & 1.73 to 3.12 & $<0.0001$ \\
\hline Pressure-related pain (yes vs no) & 2.28 & 1.59 to 3.27 & $<0.0001$ \\
\hline $\begin{array}{l}\text { Bold values indicate statistical significance. } \\
\text { Number in final model }=602 \text {. } \\
\text { `Ratio corresponds to the acceleration factor. } \\
\text { PU, pressure ulcer. }\end{array}$ & & & \\
\hline
\end{tabular}


Table 8 Skin site level analyses: multilevel logistic regression models

\begin{tabular}{|c|c|c|c|}
\hline Covariate & OR & $95 \% \mathrm{Cl}$ & p Value \\
\hline \multicolumn{4}{|l|}{ Univariable analyses } \\
\hline Age (continuous) & 1.01 & 0.99 to 1.02 & 0.4808 \\
\hline Diabetic status* (no vs yes) & 0.81 & 0.54 to 1.21 & 0.3070 \\
\hline History of weight losst (no vs yes) & 1.03 & 0.68 to 1.57 & 0.8914 \\
\hline Braden mobility ( 1 or 2 vs 3 or 4 ) & 1.14 & 0.80 to 1.64 & 0.4714 \\
\hline \multicolumn{4}{|l|}{ Skin status (reference=healthy skin) } \\
\hline Skin alterations & 6.29 & 4.21 to 9.40 & $<0.0001$ \\
\hline Category $1 \mathrm{PU}$ & 27.34 & 18.5 to 40.4 & $<0.0001$ \\
\hline Setting (hospital vs community) & 0.91 & 0.63 to 1.32 & 0.6148 \\
\hline Analgesic use (no vs yes) & 1.33 & 1.74 to 2.39 & 0.3350 \\
\hline Pressure-related pain on healthy, altered or Category 1 skin site (yes vs no) & 8.68 & 6.30 to 11.97 & $<0.0001$ \\
\hline \multicolumn{4}{|l|}{ Final multivariable model } \\
\hline \multicolumn{4}{|l|}{ Skin status (reference=healthy skin) } \\
\hline Skin alterations & 4.65 & 3.01 to 7.18 & $<0.0001$ \\
\hline Category $1 \mathrm{PU}$ & 17.30 & 11.09 to 27.00 & $<0.0001$ \\
\hline Pressure-related pain on healthy, altered or Category 1 skin site (yes vs no) & 2.25 & 1.53 to 3.29 & $<0.0001$ \\
\hline
\end{tabular}

presence of pain is a predictor for developing a category $\geq 2$ PU on the same skin site by 30 days of follow-up, after adjusting for skin status and between patient variation. Other risk factors that emerged include the presence of a category $1 \mathrm{PU}$ and alterations to intact skin at baseline, which is consistent with previous studies which have included category 1 (or equivalent and other skin status variables in multivariable modelling). ${ }^{2} 11 \quad 151819$ An unexpected finding was the high proportion of patients $(77.1 \%)$ who reported pain at baseline; while a number of these patients also had category 1 PUs, the extent of the problem was underestimated in our sample size estimate.

\section{Strengths and weaknesses of the study}

The study was designed to incorporate key quality criteria for the conduct and reporting of risk factor/prognostic factor studies ${ }^{20-28}$ to promote generalisability and minimise bias, including an a priori sample size estimate, risk factors informed by a conceptual framework, risk factor and outcome assessment by a trained clinical research team independent of the clinical team. The majority of patients received the recommended National Institute for Health \& Care Excellence (NICE) standard mattress provision of either high specification foam or alternating pressure mattresses ${ }^{29}$ and members of the research team did not alter standard care provision. Limitations of the study included a lack of blinded outcome assessment, which could have been achieved if baseline and follow-up assessments had been undertaken by two different research nurses, but there was not funding or capacity within the clinical research teams for this approach. It is feasible that they could have introduced bias to outcome assessment. The feasibility of using photography for independent blind outcome assessment is currently being determined as part of the HTA PRESSURE2 Trial (http://medhealth.leeds.ac.uk/ info/423/skin/1717/pressure_2). It is acknowledged that the patient population is not representative of the general NHS population through exclusion of patients who had cognition problems, were very sick or were terminally ill (figure 2). However, as pain is a symptom of underlying inflammation and/or nerve damage, we consider that the results are generalisable to the wider population. In addition, efforts to assess pain, soreness and discomfort should be made for all patients, including those with cognition problems using pain assessment methods established for this group of patients. ${ }^{30-32}$ It is acknowledged that patients with darkly pigmented skin were under represented in the study.

\section{Strengths and weaknesses in relation to other studies}

Building on our previous research ${ }^{11} 153334$ and in our attempts to maximise the potential event rate and so minimise the required sample size, the PU incidence rate of $25.2 \%$ was higher than predicted through inclusion of patients with evidence of acute illness and at 'high risk' of PU development. The incidence rate is comparable to other reports of category $\geq 2$ PU incidence. In our systematic review of risk factor studies, ${ }^{2} 19$ studies reported incidence rates of grade/stage $\geq 2$ ranging from $10.1 \%$ to $45.7 \%$ in heterogeneous patient populations. Of importance in terms of generalisability was our recruitment of community patients. With the exception of nursing home populations, we found only one risk factor cohort study involving community patients which reported a low incidence rate of $3.2 \%$ $(55 / 1567)$ of stage 2 PUs, but the study did not target patients with evidence of acute illness. ${ }^{2} 35$ The observed incidence rate in the community patient population was 
$26.3 \%$, although it is noteworthy that the majority of 'community' patients were recruited from rehabilitation units, with small numbers recruited in the home. In terms of risk factors identified, results are consistent with findings of the PU risk factor studies which typically identify immobility limitations, skin status and/or perfusion in multivariable analysis. ${ }^{2}$ The patient population is heterogeneous in terms of healthcare setting, but homogenous in terms of the presence of acute illness, with a high proportion of patients with diabetes $(25.4 \%)$ and an aged population (median (range) $=80$ (21-101) years) compared to other risk factor studies and indicators of high levels of comorbid disease including chronic wounds $(21.1 \%)$ and existing category $\geq 2$ PUs $(27.2 \%)$, maximising the generalisability of the study findings to those patients where primary and secondary prevention interventions require targeting (NICE 2005). It could be argued that the study should have excluded patients with existing category 1 or category $\geq 2$ PUs, but the inclusion criteria reflect the clinical population whereby patients with existing pressure injury are at risk of deterioration or PU development on other skin sites. The study is the first to undertake a skin site level analysis $;^{2}$ this type of analysis allows for assessment of risk factors at patient level, for example diabetes, and at skin site level such as presence of pain at the same skin site. In previous PU research, sample size considerations for multivariable analyses have not been used to inform study design and only 17 studies identified in a systematic review fulfilled the 'rule of thumb' sample size estimate of 10 events (or PUs) per variable in the multivariable model. ${ }^{2} 202326$ Of those only seven fulfilled key quality criteria ${ }^{20-28}$ including loss to follow-up of $<20 \%$; sufficient presentation of data to assess the adequacy of the method and analysis and a clear strategy for model building (variables included and based on a conceptual framework) and the selected model was adequate for the design. ${ }^{12} 193436-39$

\section{Meaning of the study: possible explanations and implications for clinicians and policymakers}

We have established that the presence of pain increases the risk of category $\geq 2$ PU development and accelerates time to development. This area of practice requires improved assessment, incorporation into risk assessment and treatment strategies to alleviate pain and reduce category $\geq 2 \mathrm{PU}$ development by pressure relief/reduction. Following these results, the presence of pressure-related pain has been incorporated into the PURPOSE-T a PU risk assessment framework developed as part of the PURPOSE programme (http://medhealth.leeds.ac.uk/ info/423/skin/1739/purpose_risk_assessment_framework_ raf_work_package).

\section{Unanswered questions and future research}

In our programme of work, ${ }^{4-8}$ we have established that pressure area related pain is common in hospital and community patients with intact skin areas and this study which is the first risk factor study to investigate its role suggests that pain is a factor independently predictive of subsequent category $\geq 2 \mathrm{PU}$ development. This study is the first to undertake a skin site level analysis which allowed skin site-related factors to be taken into account, however further work is required to improve the statistical methodology in this area by using more of the information collected in PU research. Further replication studies are required, and skin site level analyses should be considered in addition to patient level analyses for future PU research.

\section{Author affiliations}

${ }^{1}$ Clinical Trials Research Unit, Leeds Institute of Clinical Trials Research, University of Leeds, Leeds, UK

${ }^{2}$ Leeds Teaching Hospitals NHS Trust, Leeds, UK

${ }^{3}$ Division of Nursing, Midwifery \& Social Work, \& Central Manchester University Hospitals NHS Foundation Trust (CMFT), Faculty of Biology, Medicine and Health, University of Manchester, Manchester, UK

${ }^{4}$ School of Health \& Population Sciences, University of Birmingham, Birmingham, UK

${ }^{5}$ School of Healthcare, University of Leeds, Leeds, UK

${ }^{6}$ Leeds Institute of Clinical Trials Research, University of Leeds, Leeds, UK ${ }^{7}$ Department of Tissue Viability, Leeds Community Healthcare NHS Trust, Leeds, UK

${ }^{8}$ Mid Yorkshire Hospital NHS Trust, Wakefield, UK

Contributors JN (Professor of Tissue Viability and Clinical Trials Research) was the programme chief investigator and lead for the pain studies. She led the conception of the PURPOSE programme. She contributed to the design, protocol development, protocol implementation and interpretation of data for the pain cohort study and was involved in drafting and revising this paper critically for intellectual content. EAN (Professor of Wound Healing) was a programme coapplicant. She was involved in the conception of the PURPOSE programme; contributed to the design, protocol development, protocol implementation and interpretation of data for the pain study and was involved in reviewing this paper critically for intellectual content. SC (Programme Manager) contributed to the design, protocol development, protocol implementation and interpretation of data for the pain cohort study and reviewed the paper critically for intellectual content. DM (Patient and Public Involvement Officer) was the PPI lead for the pain cohort study and reviewed the manuscript. CD (Honorary Professor of Tissue Viability and Senior Nurse Researcher) was a programme coapplicant. She was involved in the conception of the PURPOSE programme; contributed to the design, protocol development, protocol implementation, local coordination of approvals and data acquisition for University Hospitals Birmingham NHS Foundation Trust. She was involved in the interpretation of data for the pain cohort study and reviewed the paper critically for intellectual content. MB (Professor of Nursing) was a programme coapplicant. She was involved in the conception of the PURPOSE programme; contributed to the design, protocol development, protocol implementation and interpretation of data for the pain study; contributed to drafting and revising of pain cohort study critically for intellectual content. SB (Principal Medical Statistician) was involved in the supervision of the statistical analysis and interpretation of data for the pain cohort study and contributed to drafting and revising of the paper critically for intellectual content. EMG (Tissue Viability Nurse Consultant) was a programme coapplicant. She was involved in the conception of the PURPOSE programme; contributed to the design, protocol development, protocol implementation and local coordination of approvals and data acquisition for Leeds Teaching Hospitals NHS Trust. She was involved in the interpretation of data for and contributed to the drafting of the pain cohort study paper critically for intellectual content. ILS (Medical Statistician) was involved in the statistical design and analysis and interpretation of data for the pain cohort study. She was the lead in drafting and revising the manuscript. RS (Trial 
Coordinator and Medical Student) was involved in protocol development, coordination of approvals, case report forms and database design, coordination of data acquisition and interpretation of analysis for the pain cohort study and reviewed the pain cohort paper critically for intellectual content. NS (Tissue Viability Nurse Specialist) was a programme coapplicant. She was involved in the conception of the PURPOSE programme; contributed to the design, protocol development, protocol implementation, local coordination of approvals and data acquisition for Leeds Community Healthcare NHS Trust. She was involved in the interpretation of data for the pain cohort study and reviewed the paper critically for intellectual content. LW (Programme Manager and Tissue Viability Nurse Specialist) contributed to the design, protocol development, protocol implementation and central coordination of approvals and centre set-up. She was involved in the local coordination of approvals and data acquisition for Mid Yorkshire Hospitals NHS Trust and was involved in the interpretation of data for and reviewed the pain cohort paper critically for intellectual content. JMB (Professor of Clinical Trials Research) was a programme coapplicant. She was involved in the conception of the PURPOSE programme; contributed to the design, protocol development, protocol implementation and interpretation of data for the pain cohort study. She reviewed the paper critically for intellectual content. All authors gave approval to the final version of the paper.

Funding This article presents independent research commissioned by the National Institute for Health Research (NIHR) under its programme Grants for Applied research funding scheme (RP-PG-0407-10056).

Disclaimer The views expressed in this article are those of the author(s) and not necessarily those of the NHS, the NIHR or the Department of Health.

Competing interests JN, EAN, SC and JB have received grant funding from the Worldwide Universities Network Leeds Fund for International Research Collaborations; DM has received consultancy funding from Smith \& Nephew on behalf of the Pressure Ulcer Research Service User Network for the UK (PURSUN UK) for patient and public involvement input into educational materials and JN and CD have received grant funding from Mölnlycke Healthcare. CD was a member of an expert advisory board which advises Mölnlycke on the use of dressings for pressure ulcer prevention.

Ethics approval Main Research Ethics Committee.

Provenance and peer review Not commissioned; externally peer reviewed.

Data sharing statement Requests for data should be made to the corresponding author.

Open Access This is an Open Access article distributed in accordance with the terms of the Creative Commons Attribution (CC BY 4.0) license, which permits others to distribute, remix, adapt and build upon this work, for commercial use, provided the original work is properly cited. See: http:// creativecommons.org/licenses/by/4.0/

\section{REFERENCES}

1. Gorecki C, Brown JM, Nelson EA, et al. Impact of pressure ulcers on quality of life in older patients: a systematic review. J Am Geriatr Soc 2009;57:1175-83.

2. Coleman S, Gorecki C, Nelson EA, et al. Patient risk factors for pressure ulcer development: systematic review. Int J Nurs Stud 2013;50:974-1003.

3. Girouard K, Harrison MB, VanDenKerkof E. The symptom of pain with pressure ulcers: a review of the literature. Ostomy Wound Manage 2008;54:30-40, 42.

4. Gorecki C, Closs SJ, Nixon J, et al. Patient-reported pressure ulcer pain: a mixed-methods systematic review. J Pain Symptom Manage 2011;42:443-59.

5. Pieper B, Langemo D, Cuddigan J. Pressure ulcer pain: a systematic literature review and national pressure ulcer advisory panel White paper. Ostomy Wound Manage 2009;55:16-31.

6. Nixon J, Nelson EA, Rutherford C, et al. Pressure UlceR Programme Of reSEarch (PURPOSE): using mixed methods (systematic reviews, prospective cohort, case study, consensus and psychometrics) to identify patient and organisational risk, develop a risk assessment tool and patient-reported outcome Quality of Life and Health Utility measures. Programme Grants Appl Res 2015;3(6).

7. McGinnis E, Briggs M, Collinson M, et al. Pressure ulcer related pain in community populations: a prevalence survey. BMC Nurs 2014;13:16.
8. Briggs $M$, Collinson $M$, Wilson $L$, et al. The prevalence of pain at pressure areas and pressure ulcers in hospitalised patients. $B M C$ Nurs 2013;12:19.

9. NPUAP/EPUAP. Prevention and treatment of pressure ulcers. clinical practice guideline. Washington (DC): National Pressure Ulcer Advisory Panel, 2009.

10. Bergstrom N, Braden BJ, Laguzza A, et al. The Braden Scale for predicting pressure sore risk. Nurs Res 1987;36: 205-10.

11. Nixon J, Cranny G, Iglesias C, et al. Randomised, controlled trial of alternating pressure mattresses compared with alternating pressure overlays for the prevention of pressure ulcers:

PRESSURE (pressure relieving support surfaces) trial. BMJ 2006;332:1413.

12. Defloor T, Grypdonck MF. Pressure ulcers: validation of two risk assessment scales. J Clin Nurs 2005;14:373-82.

13. Schoonhoven L, Haalboom JR, Bousema MT, et al. Prospective cohort study of routine use of risk assessment scales for prediction of pressure ulcers. BMJ 2002;325:797.

14. Machin D, Campbell MJ. Design of studies for medical research. Chichester: Wiley, 2005.

15. Nixon J, Cranny G, Bond S. Skin alterations of intact skin and risk factors associated with pressure ulcer development in surgical patients: a cohort study. Int J Nurs Stud 2007;44:655-63.

16. Coleman S, Nixon J, Keen J, et al. A new pressure ulcer conceptual framework. J Adv Nurs 2014;70:2222-34.

17. Collett D. Modelling binary data. 2 nd edn. Chapman \& Hall/CRC 2003.

18. Allman RM, Goode PS, Patrick MM, et al. Pressure ulcer risk factors among hospitalized patients with activity limitation. JAMA 1995;273:865-70.

19. Reed RL, Hepburn K, Adelson R, et al. Low serum albumin levels, confusion, and fecal incontinence: are these risk factors for pressure ulcers in mobility-impaired hospitalized adults? Gerontology 2003;49:255-9.

20. Altman DG. Systematic reviews of evaluations of prognostic variables. BMJ 2001;323:224-8.

21. Altman DG, Lausen B, Sauerbrei W, et al. Dangers of using "optimal" cutpoints in the evaluation of prognostic factors. J Natl Cancer Inst 1994;86:829-35.

22. Egger M, Smith GD, Altman DG. Systematic reviews in health care: meta analysis in context. 2nd edn. London: BMJ, 2001.

23. Harrell FE Jr, Lee KL, Matchar DB, et al. Regression models for prognostic prediction: advantages, problems, and suggested solutions. Cancer Treat Rep 1985;69:1071-7.

24. Hayden JA, Côté P, Bombardier C. Evaluation of the quality of prognosis studies in systematic reviews. Ann Intern Med 2006;144:427-37.

25. Maltoni M, Caraceni A, Brunelli C, et al. Prognostic factors in advanced cancer patients: evidence-based clinical recommendations--a study by the Steering Committee of the European Association for Palliative Care. J Clin Oncol 2005;23:6240-8.

26. Peduzzi $\mathrm{P}$, Concato J, Feinstein AR, et al. Importance of events per independent variable in proportional hazards regression analysis. II. Accuracy and precision of regression estimates. J Clin Epidemiol 1995;48:1503-10.

27. Royston P, Altman DG, Sauerbrei W. Dichotomizing continuous predictors in multiple regression: a bad idea. Stat Med 2006;25:127-41.

28. von Elm E, Altman DG, Egger M, et al. The Strengthening the Reporting of Observational Studies in Epidemiology (STROBE) statement: guidelines for reporting observational studies. Lancet 2007;370:1453-7

29. Excellence NifHaC. Pressure ulcer management: cost impact report (CG29). London, 2005.

30. Corbett A, Husebo B, Malcangio M, et al. Assessment and treatment of pain in people with dementia. Nat Rev Neurol 2012;8:264-74

31. Hadjistavropoulos T, Herr K, Turk DC, et al. An interdisciplinary expert consensus statement on assessment of pain in older persons. Clin J Pain 2007;23(Suppl):S1-43.

32. Zwakhalen SM, Hamers JP, Abu-Saad HH, et al. Pain in elderly people with severe dementia: a systematic review of behavioural pain assessment tools. BMC Geriatr 2006;6:3

33. Nixon J, McElvenny D, Mason S, et al. A sequential randomised controlled trial comparing a dry visco-elastic polymer pad and standard operating table mattress in the prevention of post-operative pressure sores. Int J Nurs Stud 1998;35:193-203

34. Nixon J, Nelson EA, Cranny G, et al. Pressure relieving support surfaces: a randomised evaluation. Health Technol Assess 2006;10: iii-iv, ix-x, 1-163. 
35. Bergquist S, Frantz R. Pressure ulcers in community-based older adults receiving home health care. Prevalence, incidence, and associated risk factors. Adv Wound Care 1999;12:339-51.

36. Schultz A, Bien M, Dumond K, et al. Etiology and incidence of pressure ulcers in surgical patients. AORN J 1999;70:434, 37-40, 43-9.

37. Bergstrom N, Braden B, Kemp M, et al. Multi-site study of incidence of pressure ulcers and the relationship between risk level, demographic characteristics, diagnoses, and prescription of preventive interventions. J Am Geriatr Soc 1996;44:22-30.

38. Brandeis GH, Ooi WL, Hossain M, et al. A longitudinal study of risk factors associated with the formation of pressure ulcers in nursing homes. J Am Geriatr Soc 1994;42:388-93.

39. Perneger TV, Rae AC, Gaspoz JM, et al. Screening for pressure ulcer risk in an acute care hospital: development of a brief bedside scale. J Clin Epidemiol 2002;55:498-504. 